\title{
Relevance of Traumatic Events and Routine Stressors at Work and PTSD Symptoms on Emergency Nurses
}

\author{
Manuel Campillo-Cruz *, José Luís González-Gutiérrez and Juan Ardoy-Cuadros (D) \\ Department of Psychology, Faculty of Health Sciences, Rey Juan Carlos University, 28922 Alcorcón, Spain; \\ joseluis.gonzalez@urjc.es (J.L.G.-G.); juan.ardoy@urjc.es (J.A.-C.) \\ * Correspondence: m.campilloc@alumnos.urjc.es or mcampillo@cop.es
}

\begin{abstract}
Emergency nurses are exposed daily to numerous stressful situations that can lead to the development of post-traumatic stress disorder (PTSD) symptoms. This study examined the relationship between traumatic events, routine stressors linked to trauma, and post-traumatic stress disorder (PTSD) symptoms in emergency nurses. For this purpose, a sample of 147 emergency nurses completed the Traumatic and Routine Stressors Scale on Emergency Nurses (TRSS-EN) and the Posttraumatic Diagnostic Scale (PDS-5). Results of correlations and moderate multiple regression analyses showed that the emotional impact of routine stressors was associated with a greater number of PTSD symptoms, and, apparently, to greater severity, in comparison to the emotional impact of traumatic events. Furthermore, the emotional impact of traumatic events acts as a moderator, changing the relationship between the emotional impact of routine stressors and PTSD symptoms, in the sense that the bigger the emotional impact of traumatic events, the bigger the relationship between the emotional impact of routine stressors and PTSD symptoms. These results suggest that the exposure to routine work-related stressors, in a context characterized by the presence of traumatic events may make emergency nurses particularly vulnerable to post-traumatic stress reactions. Some prevention measures are suggested according to the results of the study.
\end{abstract}

Keywords: emergency nursing; mental health and illness; nurses; post-traumatic stress disorder; routine stressors; traumatic events

\section{Introduction}

Emergency nurses are exposed to numerous traumatic events and routine stressors in the course of their professional activities. In this sense, traumatic events refer to shocking, frightening or dangerous experiences that can affect someone emotionally and physically $[1,2]$. In emergency care settings, traumatic events refer to shocking and disturbing experiences that nurses come across (e.g., sudden death of a child or infant) that, due to their magnitude and intensity, may threaten the nurse's ability to cope adequately [1] and may provoke strong emotional, cognitive or behavioral reactions [3]. Routine stressors refer to other, less significant, but more frequent sources of stress; however, some of them, occurring in a setting where exposure to traumatic events also occurs, seem to be related to the presence of certain manifestations of symptoms of trauma [4,5]. The majority of organizational work stressors in the emergency department can be classified as the latter [6]. Thus, situations such as work overload or conflicts with colleagues can be considered routine stressors in emergency departments.

Several studies have found clinical levels of PTSD in emergency nurses. A study carried out in Canada found that about $25.8 \%$ of a sample of Emergency Nurses met the criteria for PTSD [7]. Additionally, a Belgian study found clinical levels of PTSD in $8.5 \%$ of this population [1]. Currently, and due to the pandemic situation, several studies point to an increase in clinical levels of PTSD related to the COVID pandemic among emergency nurses [8]. In this regard, some research estimates that about $20 \%$ of emergency department staff are at an elevated risk of PTSD [9]. Other studies have reported a high 
incidence of PTSD symptoms among emergency nurses. Thus, research conducted by Iranmanesh et al. [10] in Iran showed that the majority of hospital emergency staff ( $94 \%$ of staff) reported moderate PTSD symptoms. These results are in line with those obtained by Gates et al., in the United States [11] who found that $94 \%$ of emergency nurses experienced at least one PTSD symptom after being exposed to a violent event.

Numerous studies have shown a positive relationship between traumatic events and post-traumatic symptoms [12,13]. The important role played by repeated exposure to traumatic events is also well known in the development of post-traumatic symptoms and PTSD in emergency workers such as police, first responders, firefighters and health personnel $[1,14,15]$. However, emerging evidence is indicating that repeated exposure to routine work-related stressors, is also linked to post-traumatic symptoms $[5,6,16]$. Moreover, several studies have shown that perceived uncontrollability and unpredictability of routine stressors might account for a part of such symptoms [17-19].

The role of environmental factors in the appearance of post-traumatic symptoms has been considered for many years. In fact, King and colleagues [20] highlighted the contribution of these factors to the development of PTSD symptoms in Vietnam war veterans. Their study found that exposure to certain stressful environmental settings (e.g., those dominated by daily work conflicts) were better predictors of PTSD symptoms than exposure to more severe, traumatic events (for example, the combat itself, perceived threats, or atrocities on the battlefield). Along the same lines, studies carried out with police officers show that certain routine repetitive stressors (for example, work organization problems) can play an important role in the development and maintenance of PTSD symptoms $[5,19,21]$ and, additionally, that the effects of the exposure to these routine stressors may be more severe than that of cumulative exposure to traumatic events [4]. Not many studies have been conducted among emergency professionals assessing the relationship between traumatic events, routine stressors and PTSD symptoms $[4,19,21]$. Furthermore, the results are inconclusive. In this regard, some authors have suggested that trauma severity is the only initial predictor of PTSD symptoms and that routine stressors are subsequently related to PTSD symptoms [21]. Other researchers have considered the effects of routine stressors to be independent and greater than those produced by traumatic events [4], moreover, certain studies have suggested that routine stressors mediate the relationship between traumatic events and PTSD symptoms [19]. Specifically in the field of emergency nursing, research on the relationship between traumatic events, routine stressors and PTSD symptoms has been scarce and results suggest that repeated exposure to certain routine stressors may be associated with PTSD symptoms [6]. In this regard, Laposa et al. [6] observed a significant association between one routine stressor (interpersonal conflicts at work) and PTSD symptoms; suggesting that post-traumatic effects of this routine stressor may be independent of those produced by traumatic events. These results reveal the importance of certain routine stressors in the development of PTSD symptoms, and, eventually, lead to questioning the definition of PTSD in itself (DSM-5, American Psychiatric Association, 2013), inasmuch as the DSM-5 requires the exposure to a traumatic event as a condition for diagnosing PTSD [22].

Traumatic events in an emergency setting are predictable and relatively controllable for nurses, as they are prepared for them. By contrast, continuously repeated routine situations, such as, for example, dealing with patients' relatives or interpersonal conflicts with colleagues, may be experienced by the nurses as uncontrollable and unpredictable, which may lead to sensitization to them [6]. It is well known that the uncontrollability and unpredictability of stress are consistent predictors of post-traumatic symptoms, as shown by both animal research [23] and retrospective human studies [24-26]. Thus, the results showing the harmful potential of exposure to certain routine stressors among emergency nurses with regards to PTSD symptoms [6], could be explained in terms of the sense of threat they generate, determined by the personal perception of control and the predictability of the event. Additionally, these routine stressors are not independent of traumatic events. In some cases, what makes them threatening are the possible consequences that these 
routine situations might produce in terms of quality of care and, consequently, in terms of chances of survival for the patients. In other cases, as when, dealing with patients' relatives, it often means, becoming exposed to the emotional consequences of death. Presumably, this means a greater perceived threat and, therefore, a greater source of sensitization.

In line with the above, some authors have proposed that the convergence of routine and traumatic events might be the reason why some routine stressors would reach the potential to produce post-traumatic symptoms [27]. Only a significant presence of routine stressors in a context characterized by the presence of recognizable traumatic events could provide the context for the emergence of PTSD symptoms. Along these lines, several studies show that controlling certain routine stressors associated with trauma, understood as linked to simultaneous traumatic events, can buffer the emergence and development of PTSD symptoms resulting from exposure to severe traumatic events [6,19]. Given the above, the importance of studying the role of these routine stressors as significant risk factors in interaction with traumatic events in the development of PTSD symptoms in emergency nurses is evident.

The main aims of this study are: (1) to study the association between traumatic events and routine stressors linked to trauma with regards to post-traumatic symptoms and, (2) to study the possible interaction between both types of stressors in order to predict post-traumatic symptoms [22]. Hence, the following hypotheses were proposed:

Hypothesis 1 (H1). For emergency nurses, the impact of exposure to routine stressors associated with trauma will show a greater positive association with the amount and severity of PTSD symptoms than the impact of exposure to traumatic events [6].

Hypothesis 2 (H2). Emotional impact of traumatic events will moderate the relationship between the emotional impact of routine events linked to trauma and post-traumatic symptoms. This will be reflected in an increase in the relationship between PTSD symptoms and the emotional impact of routine stressors, as the emotional intensity due to exposure to traumatic events increases [19].

\section{Materials and Methods}

\subsection{Participants}

The sample consisted of 147 emergency nurses (128 women and 19 men), working in three hospitals in Madrid (Spain) and aged between 24 and 61. The mean age of respondents was $40.41(\mathrm{SD}=8.32)$, and they had been working in the same department for a mean of 9.9 years $(\mathrm{SD}=6.69)$. In total, 111 spent more than $75 \%$ of their working time in direct contact with their patients (Table 1 presents a detailed description of the sample). Participants were required to have worked in the same department for at least one year. General exclusion criteria were: significant acute or chronic medical or psychiatric disorders.

\subsection{Instruments}

\subsubsection{Traumatic and Routine Stressors Scale on Emergency Nurses (TRSS-EN)}

The Traumatic and Routine Stressors Scale on Emergency Nurses (TRSS-EN) [28], is a 13-item measure that was used to simultaneously assess frequency (frequency of exposure) and severity (emotional impact) to common traumatic events and routine stressors associated with trauma in the daily work of emergency nurses during the previous six months. Frequency of exposure is indicated on a 7-point Likert scale, where 1 is "fewer than three times in six months" and 7 is "every day" (Frequency scale), and emotional impact of the stressful event is indicated using a second 7-point Likert scale, where 1 is "without emotional impact" and 7 is "maximum emotional impact" (Impact scale). The final scale provides six indexes: "Emotional Impact of traumatic stressors", "Emotional Impact of routine stressors", "Frequency of traumatic stressors", "Frequency of routine stressors", "Total Impact of traumatic stressors", and "Total Impact of routine stressors". 
Table 1. Basic descriptors of study participants as a function of their demographic and professional characteristics.

\begin{tabular}{|c|c|c|c|c|}
\hline Descriptive Data & Mean & SD & $n$ & Percentage \\
\hline Age $(\mathrm{N}=144)$ & 40.41 & 8.32 & & \\
\hline Children $(\mathrm{N}=147)$ & 0.99 & 1.02 & & \\
\hline Years of experience in the profession $(\mathrm{N}=147)$ & 16.48 & 7.33 & & \\
\hline Years of experience in the same job $(\mathrm{N}=147)$ & 9.9 & 6.69 & & \\
\hline \multicolumn{5}{|l|}{ Characteristics } \\
\hline Gender & & & 147 & 100 \\
\hline Male & & & 19 & 12.9 \\
\hline Female & & & 128 & 87.1 \\
\hline Relationship status & & & 145 & 100 \\
\hline In a stable relationship & & & 115 & 79.3 \\
\hline Single & & & 30 & 20.7 \\
\hline Educational level & & & 146 & 100 \\
\hline DUE & & & 88 & 60.3 \\
\hline General Nurse & & & 11 & 7.5 \\
\hline TCAE & & & 43 & 29.5 \\
\hline Others & & & 4 & 2.7 \\
\hline Employment situation & & & 147 & 100 \\
\hline Permanent & & & 72 & 49 \\
\hline Non-permanent & & & 75 & 51 \\
\hline Percentage with patients & & & 146 & 100 \\
\hline Less than $25 \%$ & & & 8 & 5.5 \\
\hline From $25 \%$ to $50 \%$ & & & 2 & 1.4 \\
\hline From $50 \%$ to $75 \%$ & & & 25 & 17.1 \\
\hline More than $75 \%$ & & & 111 & 76 \\
\hline
\end{tabular}

Note: Children refers to the number of children of the participants. DUE ("Diplomado Universitario de Enfermería") is equivalent to general nurse; TCAE ("Técnico en Cuidados Auxiliares de Enfermería") is equivalent to nursing assistant; Others (different category to the main qualification required).

The Psychometric Properties of TRSS-EN show a factorial solution with two factors for the impact scale in TRSS-EN. The eigenvalues for these factors were $>1: 6.79$ for the first and 1.57 for the second factor. The communality values ranged from 4.64 to 7.59 . According to these criteria, the factorial solution was composed of two factors. The first comprised the following items: 1 "Dealing with sudden death of young people", 2 "Dealing with death or resuscitation of a baby or young child", 3 "Handling victims of car and train crashes", 4 "Confrontation with physical trauma and burn patients", 5 "Dealing with suicide", 10 "Confrontation with child abuse and negligence" and 11 "Exposure to sudden death", with factorial weights between 0.682 and 0.846 . This factor, labeled "Traumatic stressors", was made up of traumatic events and stressors of great magnitude. Cronbach's alpha for this factor was 0.911 and Omega index was 0.857 . The second factor was composed of items 6 "Dealing with aggression, violence and threat", 7 "Inability to deliver good quality of care", 8 "Inability to help chronically ill patients", 9 "Dealing with relatives of victims/patients, 12 "Dealing with psychiatric patients", and 13 "Management of dead bodies", with factorial weights between 0.562 and 0.819 . This second factor was called "Routine stressors" and comprised events and stressors of moderate magnitude. Cronbach's alpha for this factor was 0.862 and Omega index was 0.833. Each factor (traumatic stressors and routine stressors) has 3 indexes (impact, frequency and total impact), in total six indexes. The scale has high internal consistency (Cronbach's Alpha $=0.92$ ), adequate test-retest reliability and satisfactory concurrent validity [28].

\subsubsection{Posttraumatic Diagnostic Scale for DMS-5 (PDS-5)}

The Posttraumatic Diagnostic Scale for DMS-5 (PDS-5) [29] is a 24-item measure that was used to assess PTSD symptom severity in the previous month according to DSM-5 
criteria. We used the Spanish version of the post-traumatic diagnosis scale for DSM-5. For this purpose, we used the Spanish version of the DSM-5 manual (American Psychiatric Association, 2013). The PDS-5 consists of four subscales: "Intrusion" (5 items), "avoidance" (2 items), "changes in mood and cognition" (7 Items) and "arousal and hyperreactivity" (6 Items). All these items are rated on a 5-point Likert scale from "not at all" to "6 or more times a week/severe". Four additional items ask about distress and interference caused, as well as the onset and duration of symptoms. A high score on the SA-45 represents a high level of psychiatric symptoms. Cronbach's $\alpha=0.92$, test-retest reliability $(\mathrm{r}=0.90)$. Convergent validity was deemed good [29].

\subsection{Procedure}

Before collecting data, the authors contacted several hospitals to explain the study and request their collaboration. This study was conducted in accordance with the Declaration of Helsinki [30]. The study protocol was approved by the research ethics committee of the University Rey Juan Carlos (number 030320162116).

For each hospital, we applied a simple random sampling between the whole of nursing workers from the three hospitals. We assumed a confidence level of $95 \%$ and a level of heterogeneity of $50 \%$. This sample size was enough to detect, in multiple regression models with a total number of three predictors and one tested predictor (interaction term), a medium effect size ( $\mathrm{f} 2=0.15$ ) with a target power of 0.99 , following Cohen's guidelines for small, medium, and large effects [31]. A total of 218 nursing professionals were initially involved. Thirteen participants were excluded on the application of inclusion criteria, and 68 did not return complete questionnaires (response rate $=71.7 \%$ ). The study proceeded over 16 months, from February 2017 until June 2018, during which researchers were in contact with participants. Initially, brief meetings were held in which the research aims were presented and the inclusion criteria discussed. All participants who voluntarily agreed to participate provided signed informed consent and were instructed on how to complete the questionnaire items, which were administered via the Internet. Participants were given a month to return the instruments, during which a reminder was sent by email. Data were initially collected using Google Drive. The evaluation session lasted about $20 \mathrm{~min}$. In all instances, participant anonymity was preserved by means of the use of codes.

\subsection{Data Analysis}

The analysis of the study data was performed using the statistical software IBM SPSS Statistics for Windows, version 25.0 (IBM, Armonk, NY, USA). Initially, in order to test the first hypothesis of the study, the Pearson correlations coefficients between factors of the TRSS-EN scale, and global index and subscales of the PDS- 5 test were calculated. Then, using the Student's paired $t$-test, the existence of statistically significant differences between levels of intensity of emotions aroused by traumatic events and levels of intensity generated by routine stressors in the sample were contrasted, as well as the existence of statistically significant differences between frequency of exposure to traumatic events and frequency of exposure to routine stressors. To find out whether traumatic events moderated the relationship between routine stressors and PTSD symptoms (second hypothesis of the study), a series of moderate multiple regression analyses were performed. For this, purpose, macro Process v2. 16.3 of Hayes [32]) was used, using the bootstrapping technique (10,000 bootstrap) to calculate confidence intervals (Model 1; to use this macro see Hayes, [33]) and with conditioning options: pick a point and Johnson-Neyman. The impact, frequency, and total impact of routine stressors served as independent variables, the impact of traumatic events was the moderating variable, and the different dimensions of PTSD symptoms were considered as dependent variables. The following procedure was used: each of the three routine stressor indexes (impact of routine stressors, frequency of routine stressors, and total impact of routine stressors) of the TRSS-EN scale, together with the impact of traumatic events and the five subscales of the PDS- 5 were entered separately. A total of 15 potential moderation effects were tested in this way. Statistical tests were 
carried out in a two-tailed format, all reported results were considered significant at the $p<0.05$ level.

Our research group has previously published a manuscript where the main purpose was to analyze the psychometric properties of this new scale (TRSS-EN). The aim of this study is not to analyze the validity of the scale, but rather to analyze the moderation effect of traumatic events on the relationship between routine stressors and PTSD symptoms. Although the sample is the same, the aims, statistical analysis and the main results are different according to the previous psychometric manuscript. The processing of the data carried out to meet the objective of the previous article does not interfere methodologically with this one.

\section{Results}

3.1. Associations between Impact of Traumatic Events versus Impact of Routine Stressors and PTSD Symptoms

The basic descriptive and zero-order correlations between the global TRSS-EN indices and the global PTSD index and subscales of the PDS-5 are presented in Table 2. While the emotional impact of routine stressors correlated positively both with the global index of PTSD symptoms $(r=0.307)$ and with the intrusion $(r=0.272)$, avoidance $(r=0.313)$, cognitive mood $(r=0.234)$, and arousal $(r=0.308)$ subscales, the impact of traumatic events only correlated positively with the arousal subscale $(r=0.204)$. Results from Student's paired $t$-test showed the existence of statistically significant differences between the level of emotional intensity elicited by traumatic events and the emotional intensity elicited by routine stressors $(\mathrm{t}=7.70 ; p<0.05 ; \mathrm{I}$. C $[0.54,0.91])$; additionally, they also showed the existence of statistically significant differences between the frequency of exposure to traumatic events and the frequency of exposure to routine stressors $(\mathrm{t}=-18.73 ; p<0.05 ; \mathrm{CI}$ $[-1.98,-1.60])$.

\subsection{Moderating Role of the Impact of Traumatic Events in the Association between the Impact of Routine Stressors and PTSD Symptoms}

The results of the moderate multiple regression analyses (see Table 3) showed the existence of a significant positive moderating effect of the impact of traumatic events on the relationship between the impact of routine stressors and PTSD symptoms (global index of PTSD symptoms, intrusion and arousal) and also of traumatic events in the relationship between the total impact of routine stressors and the intrusive symptoms of PTSD.

More specifically, Table 4 shows the three linear regression models, taking the global index of PTSD symptoms as the dependent variable, the impact of traumatic events as moderator, and the impact of routine stressors as the independent variable. The positive interaction effect between impact of traumatic events and routine stressors was statistically significant $(B=1.72, p<0.05, \mathrm{SE}=0.67,[0.390,3,05])$ and the model as a whole explained $16.35 \%$ of the total variance of the dependent variable $(0.1635)$, with the interaction effect only explaining $5.6 \%$ of the variance (change in $\mathrm{R} 2=0.056$ ). Likewise, the results of the pick-a-point technique showed that the conditional effect of the moderator occurred among the nurses exposed to high levels $(\mathrm{B}=7.30, p<0.01, \mathrm{SE}=1.78,[3.77,10.83])$ and means $(\mathrm{B}=4.91, p<0.01, \mathrm{SE}=1.38,[2.18,7.65])$ of traumatic event impact but not among nurses exposed to low levels $(\mathrm{B}=2.53, p<0.05, \mathrm{SE}=1.53,[-0.52,5.57])$. Furthermore, the JohnsonNeyman technique indicated the value 3.46 as the cut-off point at which the impact of traumatic events became significant, with $79.21 \%$ of the sample being above it. 
Table 2. Means, standard deviations and bivariate correlations between the six indices of the TRSS-EN scale and PTSD symptoms (PDS-5)

\begin{tabular}{|c|c|c|c|c|c|c|c|c|c|c|c|c|}
\hline & Mean & SD & 1 & 2 & 3 & 4 & 5 & 6 & 7 & 8 & 9 & 10 \\
\hline 1. Emotional impact of TE & 4.56 & 1.44 & & & & & & & & & & \\
\hline 2. Emotional impact of RE & 3.83 & 1.23 & $0.643^{* *}$ & & & & & & & & & \\
\hline 3. TE frequency & 1.98 & 0.84 & 0.004 & $0.252 * *$ & & & & & & & & \\
\hline 5. Total impact of TE & 9.02 & 4.87 & $0.541^{* *}$ & $0.547^{* *}$ & $0.809^{* *}$ & $0.476^{* *}$ & & & & & & \\
\hline 6. Total impact of RE & 15.06 & 8.23 & $0.396^{* *}$ & $0.769 * *$ & 0.503 * & $0.843^{* *}$ & $0.633 * *$ & & & & & \\
\hline 7. Global PTSD Index (PDS-5) & 12.77 & 13.40 & 0.134 & $0.307^{* *}$ & 0.084 & 0.098 & 0.138 & 0.194 & & & & \\
\hline 8. Intrusion (PDS-5) & 3.58 & 3.68 & 0.084 & $0.272 * *$ & 0.123 & 0.057 & 0.178 & 0.157 & $0.863 * *$ & & & \\
\hline 10.Cognitive mood (PDS-5) & 3.62 & 4.76 & 0.042 & 0.234 * & 0.072 & 0.128 & 0.061 & 0.174 & $0.924 * *$ & $0.678^{* *}$ & $0.671^{* *}$ & \\
\hline 11.Arousal (PDS-5) & 4.04 & 4.78 & $0.204^{*}$ & $0.308^{* *}$ & 0.053 & 0.068 & 0.144 & 0.186 & $0.934^{* *}$ & $0.713^{* *}$ & $0.680 * *$ & $0.835^{* *}$ \\
\hline
\end{tabular}


Table 3. Summary of the results of the moderate multiple regression analyzes of traumatic events as moderators in the relationship between scales of routine stressors (TRSS-EN) and PTSD symptoms (general index and scales, PDS-5).

\begin{tabular}{cccccc}
\hline & PDS-5 & Intrusion & Avoidance & Cognition & Arousal \\
\hline $\begin{array}{c}\text { Impact of } \\
\text { traumatic events }\end{array}$ & & & & \\
\hline $\begin{array}{c}\text { Impact of } \\
\text { routine stressors }\end{array}$ & $1.72^{*}$ & $0.53^{* *}$ & 0.14 & 0.41 & $0.65^{* *}$ \\
\hline $\begin{array}{c}\text { Frequency of } \\
\text { routine stressors }\end{array}$ & 0.64 & 0.25 & 0.05 & 0.78 & 0.05 \\
\hline $\begin{array}{c}\text { Total impact of } \\
\text { routine stressors }\end{array}$ & 0.20 & $0.07^{*}$ & 0.02 & 0.05 & 0.06 \\
\hline$* * 01 ; * p<0.05$. & & & &
\end{tabular}

Table 4. Complete results of moderation multiple regression analyses for the prediction of PTSD symptoms. Only significant moderation effects are presented.

\begin{tabular}{|c|c|c|c|c|}
\hline & B & SE & $\mathbf{t}$ & $\mathbf{R}^{2}$ Cor. \\
\hline \multicolumn{5}{|l|}{ Global PTSD Symptom Index (PDS-5) } \\
\hline Impact of TE & -7.52 & 2.66 & $-2.83^{* *}$ & \\
\hline Impact of RE & -3.03 & 3.18 & -0.95 & \\
\hline Impact of TE $x$ impact of RE & 1.72 & 0.67 & $2.57 *$ & $0.06^{*}$ \\
\hline \multicolumn{5}{|l|}{ Intrusion (PDS-5) } \\
\hline Impact of TE & -2.39 & 0.73 & $-3.28 * *$ & \\
\hline Impact of RE & -1.07 & 0.87 & -1.22 & \\
\hline Impact of TE $x$ impact of RE & 0.53 & 0.18 & $2.87^{* *}$ & $0.07^{* *}$ \\
\hline \multicolumn{5}{|l|}{ Arousal (PDS-5) } \\
\hline Impact of TE & -2.34 & 0.95 & $-2.46^{*}$ & \\
\hline Impact of RE & -1.57 & 1.14 & -1.38 & \\
\hline Impact of TE $x$ impact of RE & 0.65 & 0.24 & $2.70^{* *}$ & $0.06^{* *}$ \\
\hline \multicolumn{5}{|l|}{ Intrusion (PDS-5) } \\
\hline Impact of TE & -0.89 & 0.46 & -1.93 & \\
\hline Total impact of RE & -0.24 & 0.15 & -1.60 & \\
\hline Impact of TE $x$ Total impact of RE & 0.07 & 0.03 & $2.33 *$ & $0.05 *$ \\
\hline
\end{tabular}

Table 4 also shows the linear regression models, taking as the dependent variable the intrusion subscale of PTSD symptoms, the impact of traumatic events as moderator, and the impact of routine stressors as an independent variable. In this case, the coefficient of the positive interaction term between the impact of traumatic events and routine stressors was significant $(\mathrm{B}=0.53, p<0.01, \mathrm{SE}=0.18,[0.163,0.891])$ and the model in their set explained $16.60 \%$ of the total variance of the dependent variable $(0.166)$ with the interaction effect only explaining $7.1 \%$ of the variance (change in $\mathrm{R} 2=0.071$ ). Likewise, the results of the pick-a-point technique showed that the moderating effect occurred among health workers exposed to high $(\mathrm{B}=2.10, p<0.01, \mathrm{SE}=0.49,[1.13,3.06])$ and medium levels $(\mathrm{B}=1.36, p<0.01, \mathrm{SE}=0.38,[0.62,2.11])$ of traumatic event impact but not among the professionals exposed to low levels $(\mathrm{B}=0.63, p<0.05$, $\mathrm{SE}=0.42$, [ $-0.20,1.47])$. Furthermore, the Johnson-Neyman technique indicated the value 3.53 as the cut-off point at which the impact of traumatic events significant, with $78.22 \%$ of the sample being above it.

In addition, regarding the positive moderating effect of the impact of traumatic events on the relationship between the impact of routine stressors and the PTSD symptoms arousal scale, Table 4 shows that the interaction effect between the impact of traumatic events and that of routine stressors was significant $(\mathrm{B}=0.65, p<0.01, \mathrm{SE}=0.24,[0.172,1.12])$ and the model as a whole explained $15.86 \%$ of the total variance of the dependent variable (0.1586), with the interaction effect only explaining $6.3 \%$ of the variance (change in R2 $=0.0633$ ). 
Likewise, the results of the pick-a-point revealed that the moderating effect occurred among the nurses exposed to high $(\mathrm{B}=2.31, p<0.01, \mathrm{SE}=0.64,[1.05,3.58])$ and medium levels $(\mathrm{B}=1.42, p<0.01, \mathrm{SE}=0.49,[0.44,2.39])$ of traumatic event impact but not among the ones exposed to low levels $(\mathrm{B}=0.52, p<0.05, \mathrm{SE}=0.55,[-0.57,1.61])$. Additionally, the Johnson-Neyman technique indicated the value 3.94 as the cut-off point at which the impact of traumatic events became significant, with $71.29 \%$ of the sample being above it.

Finally, in relation to the positive moderating effect of traumatic events on the relationship between the total emotional impact of routine stressors and the scale of intrusion of PTSD symptoms, Table 4 shows that the interaction effect between the impact of traumatic events and the total impact of routine stressors was significant $(\mathrm{B}=0.07, p=0.05, \mathrm{SE}=0.03$, $[0.011,0.129])$ and the model as a whole explained $9.84 \%$ of the total variance of the dependent variable, (0.0984), with the interaction term alone explaining $5.08 \%$ of the variance (change in $\mathrm{R} 2=0.0508$ ). Likewise, the results of the pick-a-point technique revealed that the moderating effect occurred among the nurses exposed to high levels $(B=0.18, p<0.01$, $\mathrm{SE}=0.06,[0.06,0.29])$ of traumatic events but not among the professionals exposed to both low levels $(\mathrm{B}=-0.01, p>0.05, \mathrm{SE}=0.06,[-0.14,0.11])$ or medium levels $(\mathrm{B}=0.08, p<0.05$, $\mathrm{SE}=0.04,[-0.01,0.17])$. Furthermore, the Johnson-Neyman technique indicated the value 4.73 as the cut-off point at which the impact of traumatic events became significant, with $52.47 \%$ of the sample being above it.

There was no significant moderating effect of traumatic events on the relationship between frequency of traumatic events and PTSD symptoms, nor on the relationship between the total impact of routine stressors and PTSD symptoms. The interaction effects discussed in Table 4 can also be seen in Figure 1.
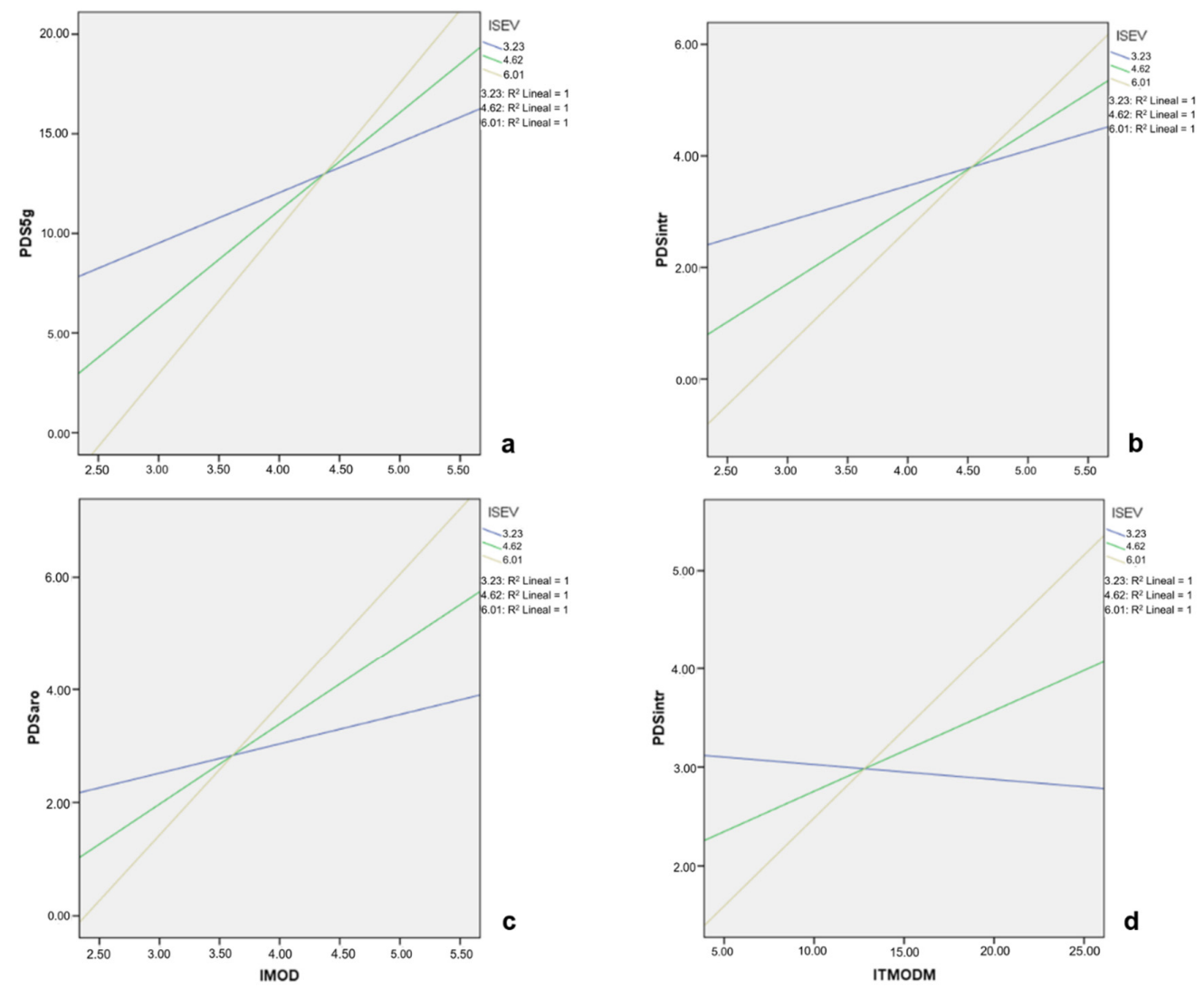

Figure 1. Graphic representation of the moderating effect of the emotional impact of traumatic events (ISEV) in the relationship between emotional impact of routine stressors (IMOD) and: (a) global index of PTSD symptoms; (b) intrusive symptoms of PTSD and; (c) PTSD arousal symptoms. In (d) the moderating effect of the emotional impact of traumatic events (ITE) on the relationship between the total impact of routine stressors (ITMODM) and the intrusive symptoms of PTSD is presented. 


\section{Discussion}

In the current study, we aimed to test two hypotheses regarding the impact of stressors and traumatic events and PTSD symptoms. The results obtained support the first hypothesis, since the emotional impact of exposure to routine stressors associated with trauma showed a stronger correlation with the amount and severity of PTSD symptoms than the emotional impact of exposure to traumatic events. These findings uphold those of Laposa et al. [6], who identified a statistically significant relationship between the impact of a specific routine stressor (interpersonal conflicts at work) and PTSD symptoms. However, we did not observe the same pattern in the relationship between traumatic events and PTSD symptoms. In this sense, traumatic events, considered in isolation, could be experienced by these professionals as less threatening since they are expected and emergency staff are prepared to cope with them. This might, likewise, facilitate the habituation to these events and consequently, lead to lower PTSD symptomatology, as has been observed among other emergency care professionals $[4,5]$.

In contrast, nurses are not specifically trained to deal with routine work stressors linked to trauma as these are perceived as uncontrollable and unpredictable. Yet when such routine stressors take place in a traumatic context, in a setting with frequent traumatic events, they become threatening. This may explain why continued exposure to routine stressors such as interpersonal conflicts at work increases vulnerability to PTSD symptomatology in emergency workers [6]. Data obtained in our study do indeed suggest that the harmful potential of routine stressors emerge in a traumatic context, where unexpected stressors which workers are not trained to cope with, are perceived as threatening.

Regarding the second hypothesis, our results also confirm that traumatic events modify the relationship between routine stressors linked to trauma and PTSD symptoms. Thus, as the emotional impact of traumatic events increases at medium (Figure 1a-c) and high levels (Figure 1a-d), so do the PTSD symptoms linked to routine stressors. The relationship between routine stressors and these symptoms is not significant unless emergency nurses are emotionally affected by the simultaneous exposure to traumatic events. These findings are in line with those of Maguen et al. [19], who highlight the importance of routine work stressors in the development of PTSD symptoms from exposure to traumatic events. They emphasize the importance of adequately managing routine work stressors in order to prevent the appearance of post-traumatic symptoms in emergency nursing.

Results obtained in the present study suggest some differences with regards to those obtained in previous research [6,19]. In this sense, the study by Laposa et al. [6], focused mainly on the analysis of a single specific routine stressor, i.e., interpersonal conflicts at work, and their results suggested the existence of a specific link between this routine stressor and PTSD symptoms. Our study, however, analyses the emotional impact of numerous typical routine work stressors in emergency nursing and our results support the idea that traumatic events could be the backbone of the deeply traumatic content of routine stressors. This point also differs from results observed by Maguen et al. [19] who suggested, in their study with police officers, that routine stressors may mediate the relationship between traumatic events and PTSD symptoms. In contrast to this mediation, which assumes the activity of routine stressors as a by-product of the previous activity of traumatic stressors, results obtained in the present study are compatible with the idea that routine stressor could be an autonomous element, able to have an effect on its own, provided that certain contextual conditions of a traumatic nature are present.

Several authors have highlighted the importance of gender differences as a vulnerability factor for the development and severity of PTSD symptoms in emergency nurses. In this regard, numerous studies conducted with emergency personnel $[4,21,34]$ were mainly comprised of men, therefore, not allowing to contrast the influence of sex on the development of PTSD. However, various results obtained in the general population show that women are at a higher risk of developing PTSD symptoms [35], furthermore, this risk is almost double that of men [36]. In contrast, in emergency nurses, the evidence has not been conclusive, since while some studies indicate that PTSD symptoms are more frequent in 
men than in women [6,37], other studies have not found this association [38], and moreover, there is also evidence that suggests that there is a higher frequency of PTSD symptoms in women [39]. In our study, with a sample of 147 emergency nurses (128 women and 19 men), we found no differences based on sex, therefore it is unclear whether, among emergency nurses, sex could increase the likelihood of developing PTSD symptoms or if these symptoms may be more severe.

In short, the present study provides evidence that, in emergency nurses, routine work stressors, occurring in a traumatic setting, may act as a trigger for the development of post-traumatic symptoms, thereby highlighting the importance of the emotional impact of the routine stressor rather than its frequency. Our results support the hypothesis that continuous exposure to traumatic events is the key to understanding how routine stressors can become so threatening as to lead to the development of post-traumatic symptoms, potentially by sensitizing in the face of routine stressors. It therefore seems advisable to refer to these stressors as routine stressors linked to traumatic settings, while noting that the presence of a traumatic context is required for these routine stressors to become threatening.

Considering the above, proper management of these routine stressors could contribute to preventing the appearance of these adverse effects. Although continuous exposure to traumatic events in emergency settings cannot be avoided, it may be possible to reduce the emotional impact resulting from staff's exposure to different routine stressors by means of better work organization or by means of specific training that provides emergency staff with the skills needed to efficiently cope with different types of stressors. In this sense, several studies point to the importance of choosing adaptive coping strategies according to the intensity and frequency of each situation to reduce such symptomatology [40] and the importance of flexibility in the use of these strategies [16].

\section{Limitations}

Some limitations of the present study should be mentioned. First, given the crosssectional nature of this study, it is important to be cautious when interpreting the direction of the associations found. Second, considering the special characteristics of emergency nurses, repeatedly exposed to traumatic events of differing emotional magnitude in their work setting on a daily basis, it is possible that the PDS-5 [29] cannot fully cover the complete spectrum of post-traumatic implications that characterize the nursing profession. In the absence of a specific questionnaire with the ability to register PTSD symptoms in this particular group of professionals, as well as for the limited correlations observed between the dimensions of TRSS-EN and PTSD symptomatology, it could be interesting to further explore this question in future research. Third, although the emotional impact of traumatic events and routine stressors is appropriately measured in the present research, and we consider it to be an essential element, it might also have been interesting to measure the perceived threat and manageability of the situation. Finally, although this study constitutes a good representation of the real distribution of women and men in the professional nursing setting, results should preferably be generalized to women. Future studies should expand this research to the male population.

\section{Conclusions}

In conclusion, we would like to highlight that our results provide a possible explanation for a phenomenon that has been observed in multiple studies published in recent years and which refers to the high correlation between non-traumatic stressors and PTSD symptoms, something that seems unlikely from a theoretical point of view. Our results, without contradicting most of these studies, show that it is possible that these correlations are due to a traumatic context in which routine stressors occur, so that repeated exposure to routine stressors in emergency settings, where traumatic stressors are common, may lead to the appearance of PTSD symptoms, which are not associated with traumatic stressors, but with routine stressors. Further research is still needed to confirm these results, as well as to understand the mechanisms by which this phenomenon, along with the intervening 
variables, occur more precisely. However, our results point to a possible integrative explanation for these conceptually anomalous phenomena that have been observed in recent years and open a path for future research.

Finally, our findings related to the relevance of routine stressors linked to traumatic environments in emergency nursing as a source of PTSD symptoms could have important implications for the prevention and care of these professionals. In this regard, while continued exposure to traumatic work events in emergency nursing cannot be avoided, it may be possible to reduce the resulting emotional impact through the application of crisis intervention techniques such as those included in the framework of a Critical Incident Stress Management program (CISM) [41-43]. Adequate management of routine stressors could also help to prevent the occurrence of the observed adverse effects and reduce the emotional impact resulting from exposure to these stressors. In this sense, psychiatric symptoms in emergency nurses could be significantly reduced and both their quality of life and their quality of care could be improved through better work organization, with adequate training plans, access to resources and support, or through specific training that equips emergency staff with the necessary skills to deal effectively with exposure to different routine stressors.

Author Contributions: Conceptualization, M.C.-C., J.A.-C. and J.L.G.-G.; Formal analysis, M.C.-C. and J.L.G.-G.; Investigation, M.C.-C., J.A.-C. and J.L.G.-G.; Methodology, M.C.-C. and J.L.G.-G.; Project administration, M.C.-C., J.A.-C. and J.L.G.-G.; Supervision, J.A.-C. and J.L.G.-G. Writingreview \& editing, M.C.-C., J.A.-C. and J.L.G.-G. All authors have read and agreed to the published version of the manuscript.

Funding: This research received no external funding.

Institutional Review Board Statement: The study was conducted according to the guidelines of the Declaration of Helsinki, and approved by the Ethics Committee of the University Review Board (protocol code 030320162116, 28 March 2016).

Informed Consent Statement: Informed consent was obtained from all subjects involved in the study. Data Availability Statement: Not applicable.

Acknowledgments: We are thankful to the emergency nursing personnel who completed the survey, the hospitals.

Conflicts of Interest: The authors declare that there is no conflict of interest.

\section{References}

1. Adriaenssens, J.; de Gucht, V.; Maes, S. The impact of traumatic events on emergency room nurses: Findings from a questionnaire survey. Int. J. Nurs. Stud. 2012, 49, 1411-1422. [CrossRef]

2. Forman-Hoffman, V.L.; Bose, J.; Batts, K.R.; Glasheen, C.; Hirsch, E.; Karg, R.S.; Huang, L.N.; Hedden, S.L. Correlates of Lifetime Exposure to One or More Potentially Traumatic Events and Subsequent Posttraumatic Stress among Adults in the United States: Results from the Mental Health Surveillance Study. In CBHSQ Data Review; Substance Abuse and Mental Health Services Administration (USA): Rockville, MD, USA, 2016; pp. 1-49.

3. Meichenbaum, D. Treating Post-Traumatic Stress Disorder. A Handbook and Practice Manual for Therapy; John Wiley \& Sons: New York, NY, USA, 1994.

4. Liberman, A.M.; Best, S.; Metzler, T.J.; Fagan, J.A.; Weiss, D.S.; Marmar, C.R. Routine occupational stress and psychological distress in police. Police Int. J. Police Strateg. Manag. 2002, 25, 421-441. [CrossRef]

5. Violanti, J.M.; Ma, C.C.; Mnatsakanova, A.; Fekedulegn, D.; Hartley, T.A.; Gu, J.K.; Andrew, M.E. Associations between Police Work Stressors and Posttraumatic Stress Disorder Symptoms: Examining the Moderating Effects of Coping. J. Police Crim. Psychol. 2018, 33, 271-282. [CrossRef] [PubMed]

6. Laposa, J.M.; Alden, L.E.; Fullerton, L.M. Work stress and posttraumatic stress disorder in ED nurses/personnel (CE). J. Emerg. Nurs. 2003, 29, 23-28. [CrossRef] [PubMed]

7. Trudgill, D.I.N.; Gorey, K.M.; Donnelly, E.A. Prevalent posttraumatic stress disorder among emergency department personnel: Rapid systematic review. Humanit. Soc. Sci. Commun. 2020, 7, 89. [CrossRef] 
8. Al Falasi, B.; Al Mazrouei, M.; Al Ali, M.; Al Dhamani, M.; Al Ali, A.; Al Kindi, M.; Dalkilinc, M.; Al Qubaisi, M.; Campos, L.A.; Al Tunaiji, H.; et al. Prevalence and Determinants of Immediate and Long-Term PTSD Consequences of Coronavirus-Related (CoV-1 and CoV-2) Pandemics among Healthcare Professionals: A Systematic Review and Meta-Analysis. Int. J. Environ. Res. Public Health 2021, 18, 2182. [CrossRef]

9. $\quad$ Rodriguez, R.M.; Montoy, J.; Hoth, K.F.; Talan, D.A.; Harland, K.K.; Eyck, P.T.; Mower, W.; Krishnadasan, A.; Santibanez, S.; Mohr, N.; et al. COVID-19-Related Stress Symptoms among Emergency Department Personnel. Ann. Emerg. Med. Adv. Online Publ. 2021. [CrossRef]

10. Iranmanesh, S.; Tirgari, B.; Bardsiri, H.S. Post-traumatic stress disorder among paramedic and hospital emergency personnel in south-east Iran. World J. Emerg. Med. 2013, 4, 26-31. [CrossRef]

11. Gates, D.; Gillespie, G.; Succop, P. Violence against nurses and its impact on stress and productivity. Nurs. Econ. 2011, 29, 59-67. [PubMed]

12. Bullman, T.A.; Kang, H.K.; Thomas, T.L. Posttraumatic stress disorder among Vietnam veterans on the Agent Orange Registry. A case-control analysis. Ann. Epidemiol. 1991, 1, 505-512. [CrossRef]

13. Friedman, M.J.; Resick, P.A.; Keane, T.M. PTSD: Twenty-five years of progress and challenges. In Handbook of PTSD: Science and Practice; Friedman, M.J., Keane, T.M., Resick, P.A., Eds.; Guilford Press: New York, NY, USA, 2007; pp. 3-18.

14. Fullerton, C.S.; Ursano, R.J.; Wang, L. Acute stress disorder, posttraumatic stress disorder, and depression in disaster or rescue workers. Am. J. Psychiatry 2004, 161, 1370-1376. [CrossRef]

15. Ward, C.L.; Lombard, C.J.; Gwebushe, N. Exposure to critical incidents in South African emergency services personnel: Prevalence and associated mental health problems. Emerg. Med. J. 2006, 23, 226-231. [CrossRef]

16. Levy-Gigi, E.; Bonanno, G.A.; Shapiro, A.R.; Richter-Levin, G.; Kéri, S.; Sheppes, G. Emotion regulatory flexibility sheds light on the elusive relationship between repeated traumatic exposure and posttraumatic stress disorder symptoms. Clin. Psychol. Sci. 2015, 4, 28-39. [CrossRef]

17. Grillon, C.; Pine, D.S.; Lissek, S.; Rabin, S.; Bonne, O.; Vythilingam, M. Increased anxiety during anticipation of unpredictable aversive stimuli in posttraumatic stress disorder but not in generalized anxiety disorder. Biol. Psychiatry 2009, 66, 47-53. [CrossRef] [PubMed]

18. Hancock, L.; Bryant, R.A. Control percibido y evitación en el estrés postraumático. Eur. J. Psychotraumatol. 2018, 9. [CrossRef]

19. Maguen, S.; Metzler, T.J.; McCaslin, S.E.; Inslicht, S.S.; Henn-Haase, C.; Neylan, T.C.; Marmar, C.R. Routine work environment stress and PTSD symptoms in police officers. J. Nerv. Ment. Dis. 2009, 197, 754-760. [CrossRef] [PubMed]

20. King, D.W.; King, L.A.; Gudanowski, D.M.; Vreven, D.L. Alternative representations of war zone stressors: Relationships to posttraumatic stress disorder in male and female Vietnam veterans. J. Abnorm. Psychol. 1995, 104, 184-195. [CrossRef]

21. Carlier, I.V.; Lamberts, R.D.; Gersons, B.P. Risk factors for posttraumatic stress symptomatology in police officers: A prospective analysis. J. Nerv. Ment. Dis. 1997, 185, 498-506. [CrossRef]

22. American Psychiatric Association. Diagnostic and Statistical Manual of Mental Disorders: DSM-5; American Psychiatric Association: Arlington, TX, USA, 2013.

23. Basoglu, M.; Mineka, S. The role of uncontrollability and unpredictability of stress in the development of post-torture stress symptoms. In Torture and Its Consequences: Current Treatment Approaches; Başoğlu, M., Ed.; Cambridge University Press: Cambridge, UK, 1992; pp. 182-225.

24. Basoglu, M.; Mineka, S.; Paker, M.; Aker, T.; Livanou, M.; Gok, S. Psychological preparedness for trauma as a protective factor in survivors of torture. Psychol. Med. 1997, 27, 1421-1433. [CrossRef]

25. Bolstad, B.; Zinbarg, R. Sexual victimization, generalized perception of control, and posttraumatic stress disorder symptom severity. J. Anxiety Disord. 1997, 11, 523-540. [CrossRef]

26. Regehr, C.; Cadell, S.; Jansen, K. Perceptions of control and long-term recovery from rape. Am. J. Orthopsychiatry 1999, 69, 110-115. [CrossRef]

27. Marmar, C.R.; Weiss, D.S.; Metzler, T.J.; Ronfeldt, H.M.; Foreman, C. Stress responses of emergency services personnel to the Loma Prieta earthquake Interstate 880 freeway collapse and control traumatic incidents. J. Trauma. Stress 1996, 9, 63-85. [CrossRef] [PubMed]

28. Campillo-Cruz, M.; González-Gutiérrez, J.L.; Ardoy-Cuadros, J.; Fernández-Muñoz, J.J. The development and exploratory psychometric properties of the traumatic and routine stressors scale in emergency room nurses (TRSS-EN). Int. J. Environ. Res. Public Health 2020, 17, 1963. [CrossRef]

29. Foa, E.B.; McLean, C.P.; Zang, Y.; Zhong, J.; Powers, M.B.; Kauffman, B.Y.; Rauch, S.; Porter, K.; Knowles, K. Psychometric properties of the posttraumatic diagnostic scale for DSM-5 (PDS-5). Psychol. Assess. 2016, 28, 1166-1171. [CrossRef]

30. World Medical Association. World Medical Association Declaration of Helsinki: Ethical principles for medical research involving human subjects. JAMA 2013, 310, 2191-2194. [CrossRef]

31. Cohen, J. Statistical Power Analysis for the Behavioral Sciences; Academic Press: New York, NY, USA, 1977.

32. Hayes, A.F. Methodology in the Social Sciences. Introduction to Mediation, Moderation, and Conditional Process Analysis: A RegressionBased Approach; Guilford Press: New York, NY, USA, 2013.

33. Hayes, A.F. Beyond Baron \& Kenny: Statistical Mediation Analysis in the New Millennium. Commun. Monogr. 2009, 76, 408-420. [CrossRef] 
34. Collins, P.A.; Gibbs, A.C. Stress in police officers: A study of the origins, prevalence and severity of stress-related symptoms within a county police force. Occup. Med. 2003, 53, 256-264. [CrossRef] [PubMed]

35. Pérez Benítez, C.I.; Vicente, B.; Zlotnick, C.; Kohn, R.; Johnson, J.; Valdivia, S.; Rioseco, P. Estudio epidemiológico de sucesos traumáticos, trastorno de estrés post-traumático y otros trastornos psiquiátricos en una muestra representativa de Chile. Salud Ment. 2009, 32, 145-153.

36. Kessler, R.C.; Sonnega, A.; Bromet, E.; Hughes, M.; Nelson, C.B. Posttraumatic stress disorder in the National Comorbidity Survey. Arch. Gen. Psychiatry 1995, 52, 1048-1060. [CrossRef]

37. Hosseininejad, S.M.; Jahanian, F.; Elyasi, F.; Mokhtari, H.; Koulaei, M.E.; Pashaei, S.M. La prevalencia del trastorno de estrés postraumático entre las enfermeras de emergencia: Un estudio transversal en el norte de Irán. BioMedicine 2019, 9, 19. [CrossRef]

38. Narimani, M.Z.A.; Basharpoor, S. Prevalence of posttraumatic stress disorder in hospital emergency nurses and fire department workers in Uremia city. J. Res. Behav. Sci. 2010, 8, 69-74.

39. D’Ettorre, G.; Ceccarelli, G.; Santinelli, L.; Vassalini, P.; Innocenti, G.P.; Alessandri, F.; Koukopoulos, A.E.; Russo, A.; d’Ettorre, G.; Tarsitani, L. Post-Traumatic Stress Symptoms in Healthcare Workers Dealing with the COVID-19 Pandemic: A Systematic Review. Int. J. Environ. Res. Public Health 2021, 18, 601. [CrossRef]

40. Sheppes, G.; Scheibe, S.; Suri, G.; Radu, P.; Blechert, J.; Gross, J.J. Emotion regulation choice: A conceptual framework and supporting evidence. J. Exp. Psychol. Gen. 2014, 143, 163-181. [CrossRef] [PubMed]

41. Mitchell, J.T. When disaster strikes. The Critical Incident Stress Debriefing process. J. Emerg. Med. Serv. 1983, 8, 36-39.

42. Cudmore, J. Preventing post traumatic stress disorder in accident and emergency nursing. A review of the literature. Nurs. Crit. Care 1996, 1, 120-126.

43. Morrow, H.E. Coordinating a multiple casualty Critical Incident Stress Management (CISM) response within a medical/surgical hospital setting. Int. J. Emerg. Ment. Health 2001, 3, 27-34. 